\title{
The VLTI as a tool to study eclipsing binaries for an improved distance scale
}

\author{
K. Shabun ${ }^{1}$ A. Richichi ${ }^{1}$ U. Munari ${ }^{2}$ A. Siviero ${ }^{2}$ and B. Paczynski ${ }^{\dagger}$ \\ ${ }^{1}$ ESO, European Southern Observatory \\ email: kshabun@eso.org \\ ${ }^{2}$ INAF, Osservatorio Astronomico di Padova
}

\begin{abstract}
Long-baseline interferometry with facilities such as the ESO VLTI is beginning to have the capability to measure directly in the range of milliarcsecond and less the angular separation and the angular diameter of some selected eclipsing binary systems. We have begun to carry out such observations with the AMBER instrument. In the special case of double-lined eclipsing binaries with well-detached components, from radial velocity and light curves it is possible to obtain a full solution of all orbital and stellar parameters, with the exception of the effective temperature of one star, which is normally estimated from spectral type or derived from atmospheric analysis of the spectrum or reddening-corrected photometric colors. In particular, we aim at deriving directly the effective temperature at least of one component in the proposed system, thereby avoiding any assumptions in the global solution through the Wilson-Devinney method. We have obtained an independent check of the results of this method concerning the distance to the system. This represents the first step toward a global calibration of eclipsing binaries as distance indicators. Our results will also contribute to the effective temperature scale for hot stars. The extension of this approach to a wider sample of eclipsing binaries could provide an independent method to assess the distance to the LMC.
\end{abstract}

Keywords. techniques: interferometric, binaries: eclipsing, stars: distances, stars: fundamental parameters, Magellanic Clouds

\section{Introduction}

We are using AMBER at the VLTI, currently offered on UTs in JHK bands, in the combination that provides the highest angular resolution, i.e. triplets which include the UT1-UT4 baseline and the low-resolution LR-HK mode. This mode provides dispersed visibilities over the $1.5-2.4$ micron range.

Our goal is to measure a restricted number of EB systems with a (semi-major axis) in the range $1-5$ mas, and angular diameters $\theta_{1}, \theta_{2}$ to $0.4-1.2$ mas. This will be accomplished by observing each system at least four times, at a precise separated phase of the orbital period $P$ corresponding to the maximum separation. Time has been granted to observe $\delta$ Ori, $a=1.4, \theta_{1}=0.98, \theta_{2}=0.55, P=5.73 \mathrm{~d}$ (distance $280 \mathrm{pc}$ ), in the ESO Period 78. Unfortunately only two observational runs have been successfully accomplished in December 2006 and March 2007, the rest two runs were deferred to Period 80. Also four additional observations of $\delta$ Ori has been granted for the Period 80 , as is the time for observations of $\eta$ Ori, $a=1.7, \theta_{1}=0.84, \theta_{2}=0.7, P=7.98 \mathrm{~d}$ and R CMa, $a=1.1$, $\theta_{1}=0.65, \theta_{2}=0.5, P=1.13 \mathrm{~d}$.

\section{Overview}

Each observation gives us 3 visibilities and one closure phase for the system. These values allow us to determine the separation and the orientation of the system. We observe 


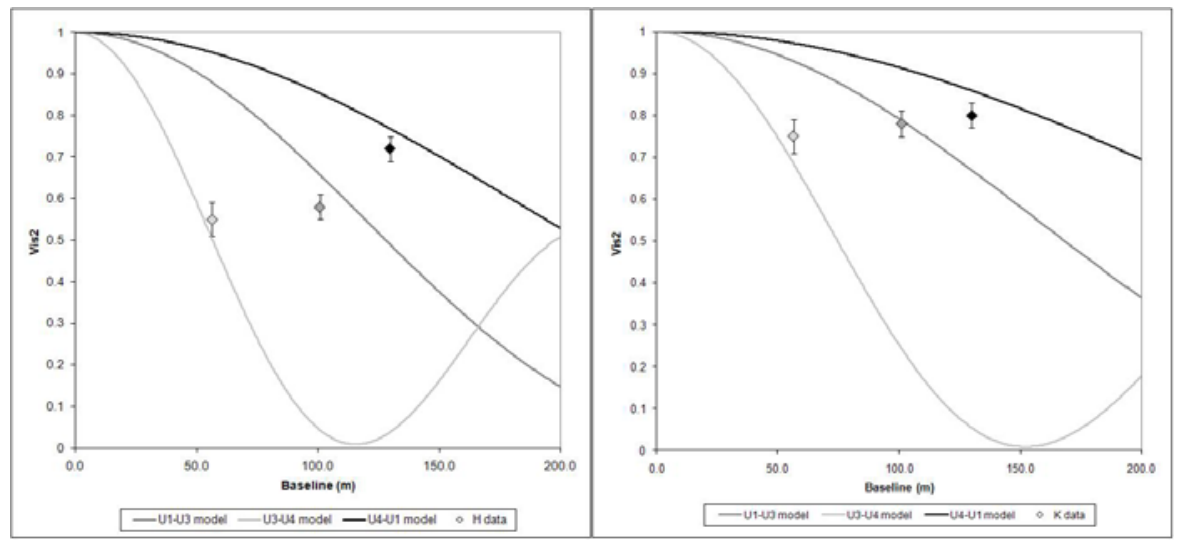

Figure 1. Averaged H- and K-band squared visibilities of $\delta$ Ori, observed on 31.12.2006 with AMBER UT1-UT3-UT4 (dots). The model fit (curves): separation 1.9 mas, position angle 75 degrees, diameters of 0.9 and 0.6 mas respectively. Each dot/curve color corresponds to a different baseline.

the systems at the time of maximum separation, at the opposite orbital phases. The figure 1 shows the averaged squared visibilities of $\delta$ Ori observed by AMBER at 31.12.06. The symbols are the visibilities averaged over the wavelengths, H-band 1.72 and K-band 2.26 microns, in reality we have 16 channels in the $\mathrm{K}$ and 11 channels in $\mathrm{H}$ band. The solid curves in both figures represent the (preliminary) best fit to the data of the separation and position angle of the binary, with respect to the length and position angle of the baselines. The figures show a good match between the model and observed visibilities. Not all parameters used in the fit are available from the literature, and in addition there is a wide scatter of values. However, we note a general agreement with the results of our preliminary fit.

The high quality radial velocity and photometric light-curves for the proposed targets are currently being collected at Asiago Observatory. A total of 49 high resolution Asiago Echelle spectra of $\delta$ Ori have already been collected in 10 different nights during the time interval of ESO Period 78. The spectra cover the range from 3800 to $7300 \AA$ at a resolving power 30000 . We have achieved a $\mathrm{S} / \mathrm{N}$ per pixel on the extracted spectrum in excess of 150 . In addition, accurate $B, V$ photoelectric photometry has been collected in 12 separate nights to map out the lightcurve of an eclipse.

We stress that this observational breakthrough is made possible for the first time by a combination of the long VLTI baselines with the accuracy, sensitivity and wavelength range of the UT+AMBER. Full solutions (masses, radii, effective temperatures, distances) will be obtained for the proposed EBs after the completion of the full observational program. If our initial observations are successful, we plan to expand them to a wider sample of binaries with diverse spectral types. One of the possible applications of the completed calibration is the precise determination of the distance to the Large Magellanic Cloud.

\section{References}

Paczynski, B. 1997, Space Tel. Sc. Inst. p. 273

Munari, U., Siviero, A. et al. 2004, A\&A 418, L31

Siviero A., Munari U. et al. 2004, A\&A 417, 1083

Richichi, A. et al. 2005, A\&SA 431, 773 